\title{
Guselkumab for the Treatment of Palmoplantar Pustulosis: A Japanese Perspective
}

\author{
Toshiyuki Yamamoto \\ Department of Dermatology, Fukushima \\ Medical University, Fukushima, 960-1295, \\ Japan
}

\begin{abstract}
Palmoplantar pustulosis (PPP) is a chronic inflammatory disorder characterized by sterile pustules predominantly involving the palms and soles. PPP is refractory to various therapies such as topical ointment, oral medicine, and phototherapies. Pustulotic arthroosteitis (PAO) is a major comorbidity of PPP that severely impairs patients' quality of life. Recently, guselkumab, a monoclonal antibody against IL-23, has been available for the treatment of PPP in Japan. The purpose of the present review is to describe the characteristics of Japanese PPP patients and biologic therapy of PPP/PAO using guselkumab. Most Japanese dermatologists consider PPP as a distinct entity and co-existence of PPP and psoriasis is rare. However, outside Japan, PPP is often considered to be palmoplantar psoriasis, and extrapalmoplantar lesions associated with PPP are regarded as psoriasis. PPP develops or exacerbates either with or without arthralgia, following focal infections, such as tonsillitis, odontogenic infection, and sinusitis. Treatment of focal infection results in dramatic effects on cutaneous lesions as well as joint pain. By contrast, we sometimes see patients whose skin/ joint symptoms do not improve after treatment of focal infection, whose focus of infection cannot be identified even in a detailed examination, and/or who refuse tonsillectomy even if strongly recommended. Such cases are considered to be indications of biologics. In this review, clinical features, pathophysiology and guselkumab therapy are discussed.
\end{abstract}

Keywords: palmoplantar pustulosis, pustulotic arthro-osteitis, therapy, biologics

\section{Introduction}

Palmoplantar pustulosis (PPP) is characterized by multiple aseptic small pustules, as well as vesiculopustules, scales, crusts, and erythemas, predominantly involving the palms and soles. Some regard PPP as an acral variant of pustular psoriasis, while others consider PPP to be a distinct entity, different from psoriasis. ${ }^{1-7}$ Many aspects of PPP resemble those of psoriasis, and shared pathogenesis has been suggested between PPP and psoriasis, either plaque-type or pustular-type. ${ }^{8}$ One of the important differences between PPP and psoriasis is that PPP is closely related to focal infection and thus treatment of ie tonsillar and dental infection is preferentially required. However, some patients develop PPP without having focal infection. Recently, biologic targeting IL-23 has been available for the treatment of PPP in Japan. This review addresses the characteristics of Japanese patients with PPP, and also discusses current therapies using biologics.

\section{Clinical Features}

The initial lesions of PPP are vesicles, which turn into vesiculopustules (pustulovesicles), and then rapidly become purulent. ${ }^{9}$ Typical clinical features present with small 
pustules, scales, brownish crusts, and ill-circumscribed erythemas on the palms and soles. Although palms and soles are usually affected bilaterally, cases with unilateral involvement have been rarely observed (Figure 1A). During the long-term course, the phenotype of plantar PPP sometimes changes to keratotic lesions devoid of pustules, mimicking psoriasis (Figure 1B).

PPP lesions sometimes develop beyond the palms and soles, presenting with thin, scaly erythemas, on extrapalmoplantar areas such as the forearms, elbows, dorsa of the feet, lower legs, knees, and buttocks. ${ }^{5}$ Such lesions clinically resemble psoriasis, nummular eczema, and/or asteatotic eczema. Many Japanese dermatologists consider those scaly erythemas to be extra-palmoplantar lesions associated with PPP, ${ }^{5}$ which are different from psoriasis; while those lesions may be considered as psoriasis in other countries. ${ }^{10,11}$ There are two kinds of extra-palmoplantar lesions; one is psoriasiform scaly erythematous lesions (chronic type) and the other is pustular lesions (acute type) (Figure 1C and D). Infiltration of the scaly erythema is mild and the borders are ill-defined as compared with plaque-type psoriasis. The histological features are mild acanthosis with focal parakeratosis and exocytosis, while unlike psoriasis, Munro's microabscess is uncommon, and dilation of the capillaries in the papillary dermis is absent. ${ }^{5}$ By contrast, a biopsy specimen taken from a pustular lesion reveals Kogoj's spongiform abscesses.

Nail changes were seen in one third of the PPP patients. ${ }^{12,13}$ A previous study from Europe showed that subungual pustules to be most commonly seen lesions (67\%) and onycholysis and pitting were observed in almost $40 \%$ of patients. ${ }^{12}$ By contrast, another Japanese study demonstrated that onycholysis was most frequently detected $(50 \%)$, whereas subungual pustules were rarely observed $(14 \%){ }^{13}$ Such differences may be due to the differences in patients' population (including or excluding psoriasis), or genetic background. In comparison with psoriasis, PPP patients with nail deformity rarely complain of distal interphalangeal (DIP) joint pain [manuscript in preparation]. Nail lesions are refractory to topical therapies, and treatment of focal infection results in marked improvement of nail lesions (Figure 2). Alternatively, biologics may be expected for PPP nails.

\section{Diagnosis}

In the majority of cases, clinical manifestation presents with typical features such as small pustules, scales, and erythema on the palms and/or soles, and thus biopsy is not
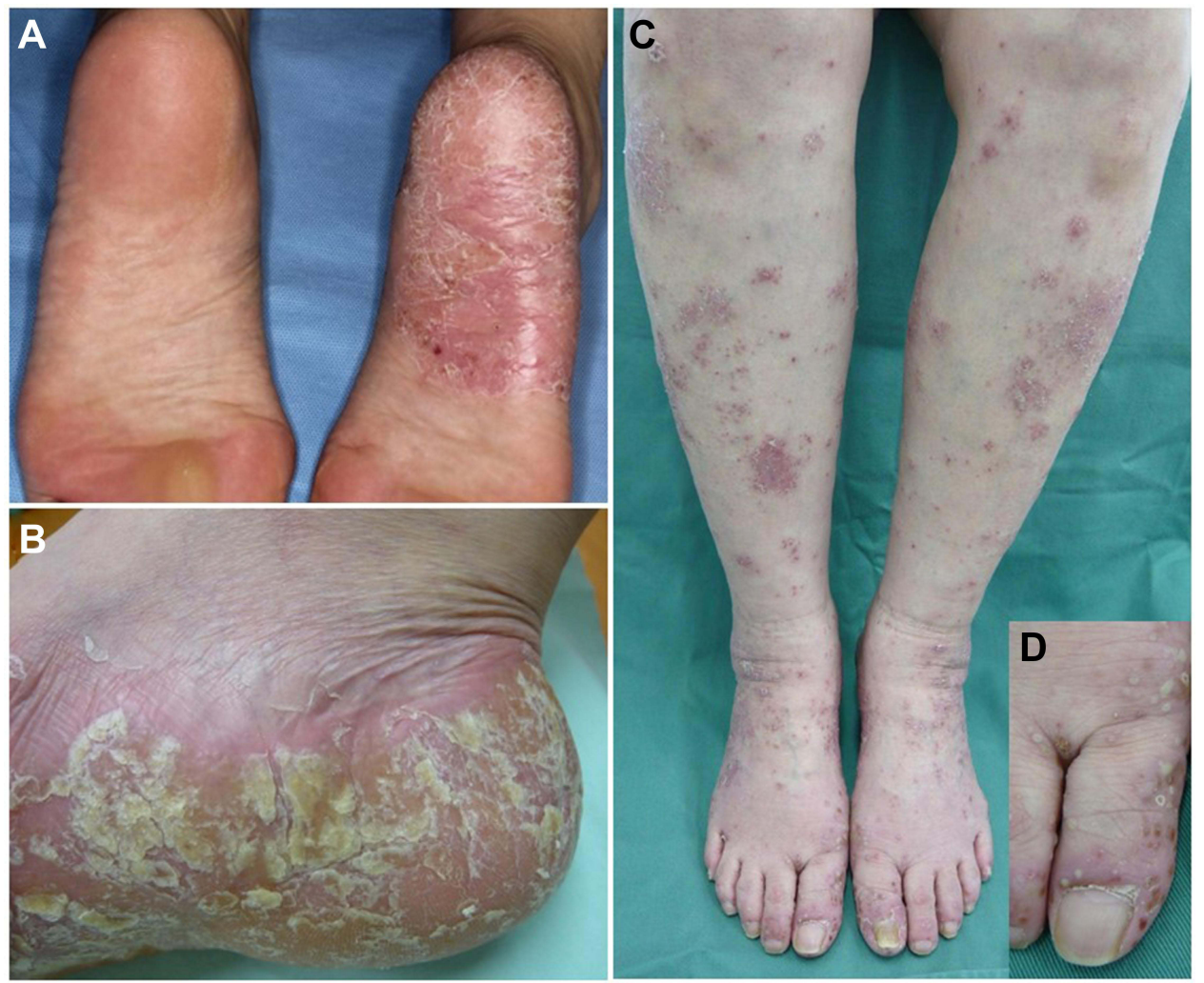

Figure I Atypical features of PPP. (A) Unilateral plantar involvement. (B) Plantar hyperkeratosis and erythematous lesions. (C) A number of ill-defined erythematous lesions with scales on the lower extremities. (D) Small pustular lesions. 

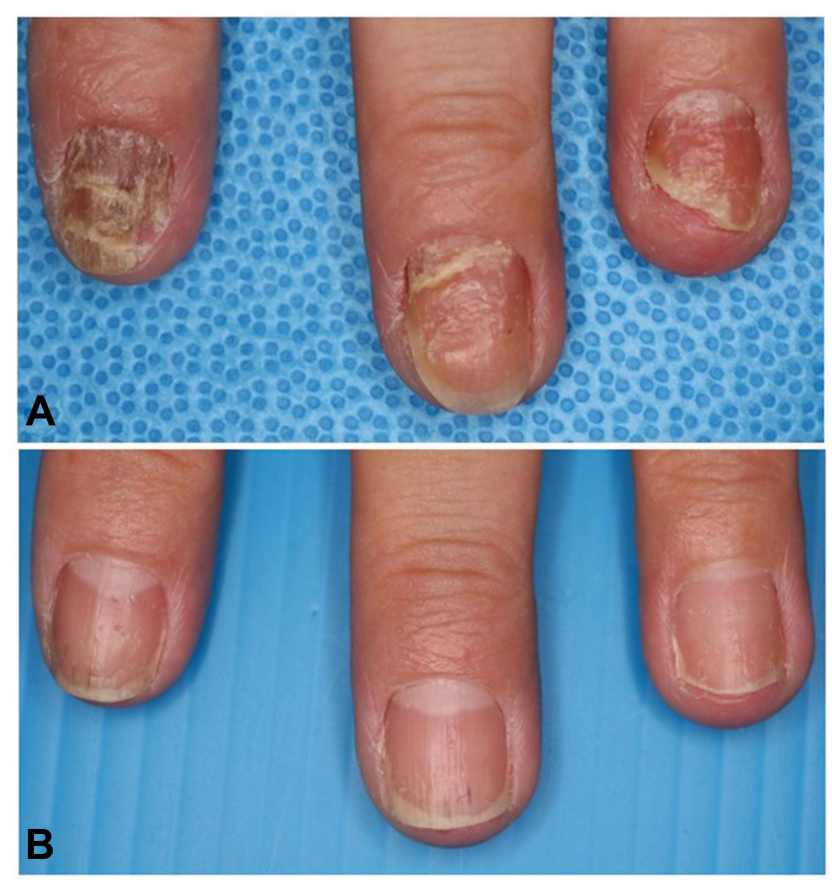

Figure 2 Improvement of PPP nail after tonsillectomy. Before (A) and I year after tonsillectomy (B).

always performed in the diagnosis of PPP. By contrast, if pustules are not detected when patients present to us, the diagnosis is sometimes difficult and careful follow-up is required. The characteristic histopathological features of PPP exhibit intraepidermal infiltration of polymorphonuclear neutrophils. Pustules are formed from vesicles and detailed analysis depending on the phases of vesicles, pustulovesicles, and pustules, showed several steps such as vesicles reaching the stratum corneum without spongiosis, vesicles including some monocytes and neutrophils, neutrophil infiltration into the epidermis beneath vesicles, microabscesses on the edges of vesicles, and vesicles filled with abundant monocytes and neutrophils. ${ }^{14}$

Differential diagnosis includes several diseases such as pompholyx, dyshidrotic eczema, contact dermatitis, hand eczema, fungal infection, palmoplantar psoriasis, and pustular psoriasis, as well as rare diseases such as lichen planus, pityriasis rubra pilaris, mycosis fungoides, and eosinophilic pustular folliculitis involving the palms/soles (Figure 3). Acropustulosis continua of Hallopeau (ACH) is a variant of localized pustular psoriasis, and is characterized by chronic sterile pustules around and under the nail; however, the patients do not have palmoplantar lesions. Acute generalized pustular bacterid (AGPB), or pustulosis acuta generalisata, is most frequently triggered by streptococcal infection, and presents with scaly erythemas and pustules widely spreading to the trunk and extremities,
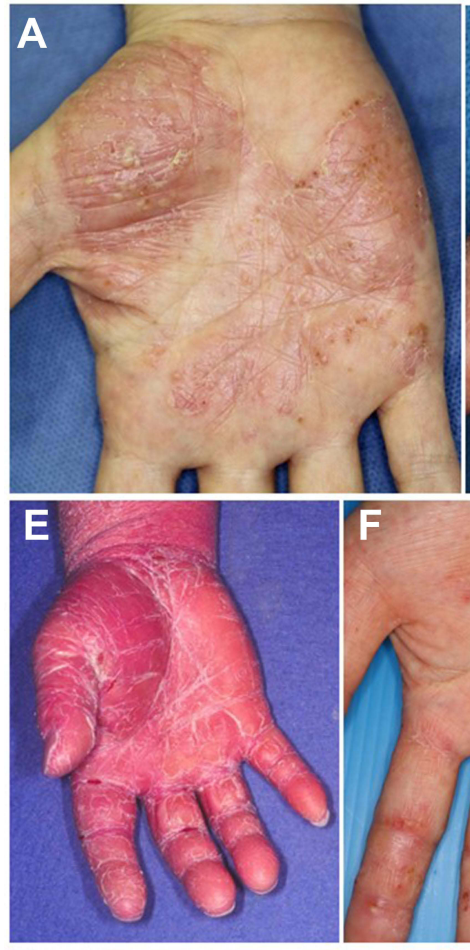
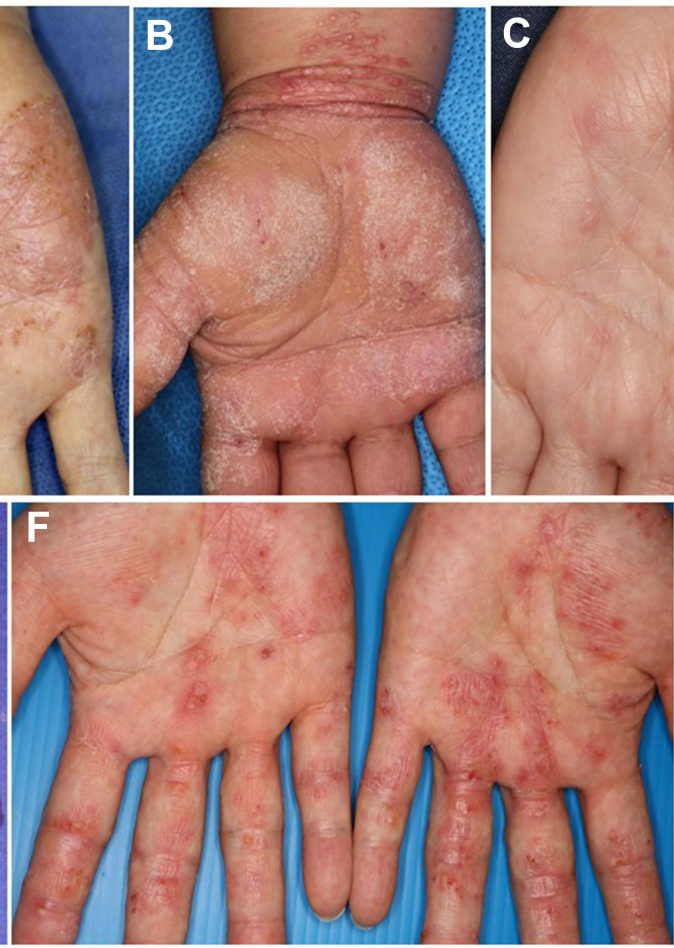
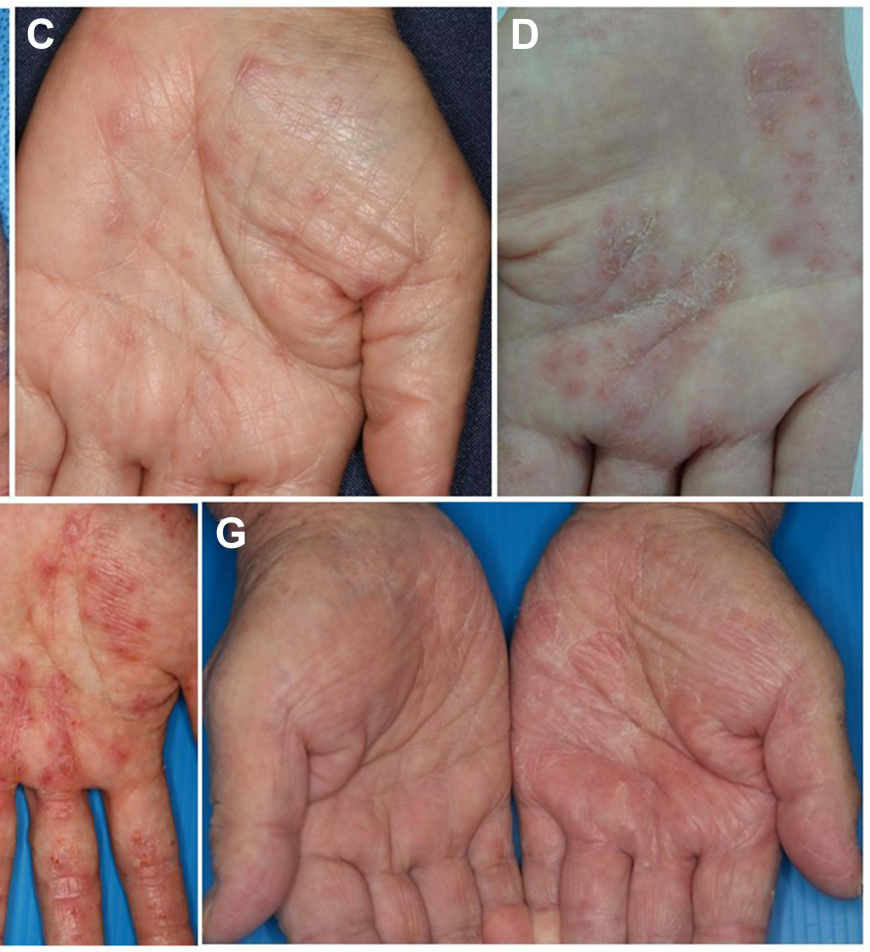

Figure 3 Various features affecting palms. (A) PPP, (B) psoriasis, (C) lichen planus, (D) dyshidrotic eczema, (E) pityriasis rubra pilaris, (F) contact dermatitis, (G) tinea manus. 
accompanied by general symptoms such as fever, myalgia, and arthralgia. Thus, AGPB is sometimes difficult to differentiate from PPP.

\section{Joint Manifestation}

PAO is the major comorbidity of PPP, affecting the clavicles, sternum, and sternoclavicular joints, as well as peripheral joints. ${ }^{15}$ PAO is also triggered by focal infection. Previous studies have shown that the ratio of PAO is around 20 to $30 \%$ of PPP patients. ${ }^{16-18}$ In a recent multicenter study, we showed that PAO was observed in $28.6 \%$ (165/576) of Japanese PPP patients, with a female predominance (male-to-female ratio; 1:3.7). The mean disease duration of PAO was 6.0 years, and smoking habit was observed in 104 patients. Focal infection was detected in 74 patients, among whom tonsillar infection $(n=41)$, sinusitis (8), odontogenic infection (40), and others (2) were observed. Fifteen patients had multifocal infection. Technetium bone scintigraphy revealed that increased uptake was most frequently observed in the sternocostoclavicular region, followed by the wrist and ankle, sacroiliac joint, knee and elbow, finger and toe, lumbar spine, thoracic spine, scapula, spine, and thigh. ${ }^{19}$

\section{Focal Infection}

PPP is occasionally worsened by bacterial infection, ie tonsillitis, sinusitis, and dental infection, which initially triggers innate immune response, followed by acquired immune activation via toll-like receptors. ${ }^{20}$ Bacterial products stimulate enhanced production of IL-23, which triggers T-cells to produce IL-17. Th17 cells express CCR6 and produce CCL20. ${ }^{21}$ Expression of CCL20 is upregulated in the PPP lesional skin, suggesting an important interaction between CCL20/CCR6. Tonsillitis is the representative focal infection associated with PPP. Stimulation with $\alpha$-streptococcal antigens also enhances chemokine receptor CCR6 expression in tonsillar T-cells, ${ }^{22}$ which may attract CCL20-positive T-cells. Bacterial infection activates tonsillar T-cells to enhance cutaneous lymphocyte-associated antigen (CLA) expression, ${ }^{23}$ which leads to migration into the skin and joints. ${ }^{15,24}$ In addition, cytokine production such as IL-6, TNF- $\alpha$, and interferon$\gamma\left(\right.$ IFN- $\gamma$ ) is enhanced. ${ }^{25}$ Tonsillectomy results in improvement and even completely relieves cutaneous and/or skeletal lesions. According to a retrospective study analyzing a number of cases, skin lesions disappeared in $67 \% 24$ months later, and joint pain disappeared in $83 \% 12$ months later. $^{26}$ These findings strongly suggest that tonsillar infection plays a key triggering role to lead to a sequential event in PPP.

A number of cytokines induce inflammation in the joints and osteoproliferation in the bones. Especially, IL17 and IL-22 secretion are upregulated following infection. $^{27}$ IL-22 regulates human mesenchymal stem cell function, including proliferation, migration, and osteogenesis, ${ }^{28}$ suggesting a possible physiological role in PAO. Moreover, Wnt family proteins and $\beta$-catenin pathway regulate bone remodeling, and activation of Wnt/ $\beta$-catenin signaling induces osteoblastogenesis. ${ }^{29}$ Bone morphogenetic protein (BMP) families are also possible mediators, and transforming growth factor- $\beta$ (TGF$\beta$ )/BMP signaling plays a critical regulatory role in new bone formation. ${ }^{30}$

Other than tonsillectomy, PPP improves after dental treatment in many cases. A recent study reported that $63 \%$ of the 70 PPP patients showed positive clinical outcome after dental infection control. ${ }^{31}$ In periodontitis, the bacterial microenvironment with the prominence of Porphyromonas gingivalis induces IL-17A production, which may play a role in the inflammatory process of PPP. $^{32}$ In addition, sinusitis, cholecystitis, and appendicitis also sometimes precede the onset of PPP.

\section{Pathophysiology}

In PPP, selective accumulation of neutrophils in the epidermis may be caused by the local generation of neutrophil-specific chemoattractants, including IL-8, growthrelated oncogene- $\alpha$ (GRO- $\alpha$ ), and complements. In addition, IL-17 promotes neutrophil migration via the release of CXC chemokines. Early-stage vesicles of PPP contain a number of mononuclear cells expressing proteinase-3, which is responsible for processing mature cathelicidin, ${ }^{33}$ and increased levels of cathelicidin upregulate the expression of IL- $1 \alpha$, IL-1 $\beta$, IL-8, and IL-17C in keratinocytes. ${ }^{34}$ IL-8 protein levels are increased in the exudates of PPP lesions, ${ }^{35}$ and IL- 8 expression was detected in the epidermis of PPP by immunohistochemistry, ${ }^{36}$ suggesting that lesional keratinocytes may play an important role in eliciting neutrophilic inflammatory response mechanisms. Additionally, a number of CD4-positive T-cells infiltrate below and around the neutrophilic abscess, which suggests an important role in the pathogenesis of PPP by releasing inflammatory cytokines. ${ }^{37}$

Acrosyringium in the palms/soles is reported to be the primary target for inflammation in PPP. ${ }^{38,39}$ The sweat gland apparatus is a neuroendocrine organ expressing 
several neuroendocrine markers. Nicotinic acetylcholine receptors (nAChRs) are expressed in the eccrine glands, ducts and endothelium in the skin, whose patterns are altered by smoking. ${ }^{40}$ Nicotine acts on nAChRs as an agonist, among which $\alpha 7 \mathrm{nAChR}$ is expressed in epithelial cells. Epidermal $\alpha 7 \mathrm{nAChR}$ expression was abolished in PPP skin, compared with normal skin. ${ }^{41}$ Thus, PPP patients may be incapable of activating the endogenous nicotinic anti-inflammatory pathway. Moreover, cigarette smoke exerts various effects and cultured tonsillar epithelial cells from PPP patients secreted IL-36 by exposure to cigarette smoke extract. ${ }^{42}$

The IL-23/IL-17 inflammatory pathway has recently been suggested to be important in PPP. IL-23 as well as IL-23 receptor expression is upregulated, ${ }^{43,44}$ and IL-17 is detected close to or in the acrosyringium, ${ }^{45}$ in PPP lesional skin. IL-23 is mainly produced by activated myeloid cells such as macrophages and dendritic cells, and neutrophils and keratinocytes are also potential sources. IL-23 is important in the regulation of IL-17 producing cells, and plays an orchestrating role as master regulator in the pathogenesis psoriasis, psoriatic arthritis, and inflammatory bowel diseases. IL-23 promotes the differentiation of osteoclasts indirectly via the induction of Th17 cell polarization and IL-17A production, whereas the direct effects of IL-23 on osteoclastogenesis are less well understood.

\section{Therapy by Biologics}

Previously, ustekinumab was found to be an effective and safe therapy for refractory cases of PPP, although the examined number was small. ${ }^{46}$ Positive responses were initially obtained 2 to 3 weeks after initial administration, and complete resolution was achieved at week 20. By contrast, another study did not show a sufficient effect of ustekinumab in four cases of PPP. ${ }^{47}$ Furthermore, combination therapy using adalimumab and ustekinumab has been reported in a severe case. ${ }^{48}$ PPP or PPP-like lesions are sometimes induced, which is called paradoxical reaction, during treatment with TNF inhibitors, ${ }^{49}$ and also other biologics such as ustekinumab ${ }^{50}$ and secukinumab. ${ }^{51}$ However, judging from the case series and also single case reports, biologics may be expected as a new effective therapy for PPP, especially in cases accompanying PAO.

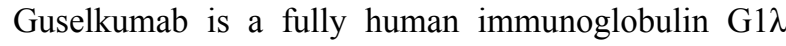
monoclonal antibody that selectively binds to the p19 subunit of IL-23. The clinical efficacy of subcutaneous injection of guselkumab in the treatment of moderate to severe PPP that did not respond adequately to conventional treatments was assessed in a randomized doubleblind placebo-controlled clinical trial. ${ }^{52}$ The patients were over 20 years of age, and suffered from moderate to severe PPP with an inadequate response to prior conventional treatment with topical corticosteroids, vitamin D3 analogues, etretinate and phototherapy. Baseline demographics were generally similar between the guselkumab $(n=25)$ and placebo $(n=24)$ groups, with a score of $\geq 7$ on the palmoplantar pustulosis severity score (PPSI). At week 16, PPP area and severity index (PPPASI) scores were significantly improved, and the proportion of patients achieving 50\% reduction of PPPASI scores were significantly increased. Improvement of efficacy scores was maintained through 24 weeks in the guselkumab group. Regarding biomarkers, a significant reduction from baseline in circulating IL-17A levels was observed at weeks 4 and 16 by guselkumab treatment, whereas no significant changes were observed in the placebo group.

In the Phase 3 trial, 159 patients $\geq 20$ years of age with PPP for 24 or more weeks before screening who had an inadequate response to conventional therapies were enrolled. ${ }^{53}$ The patients were randomly assigned to receive either guselkumab (100 mg) $(\mathrm{n}=54)$, guselkumab (200 mg) $(n=52)$ or placebo $(n=53)$. Both guselkumab groups met primary endpoint at week 16, and showed a significant improvement in the changes of PPPASI scores. A significantly higher proportion of the patients in the guselkumab 100-mg group achieved a PPPASI 50 response at week 16, and such efficacy was consistent through week 52. Health-related quality of life assessed by Dermatology Life Quality Index (DLQI) score also improved. In Japan, more than 10 biologics including guselkumab are used for psoriasis therapy, but guselkumab is currently the only biologic approved for PPP. The representative photographs before and after guselkumab therapy are shown in Figure 4.

In another study, 66 patients with PAO were randomized to receive either guselkumab (100 mg or $200 \mathrm{mg}$ ) or placebo. Efficacy was evaluated EQ-5D pain/discomfort dimension score, and classified into 5 levels of absent, mild, moderate, severe, and extreme. ${ }^{54}$ As an objective index, magnetic resonance imaging was used. The proportions with an EQ-5D pain/discomfort dimension score of no or slight pain/discomfort in the guselkumab group increased from baseline (33.3\%) to weeks 52 (87.5\%). At week 16, higher proportion of patients with no or slight pain/discomfort was observed in the guselkumab group 

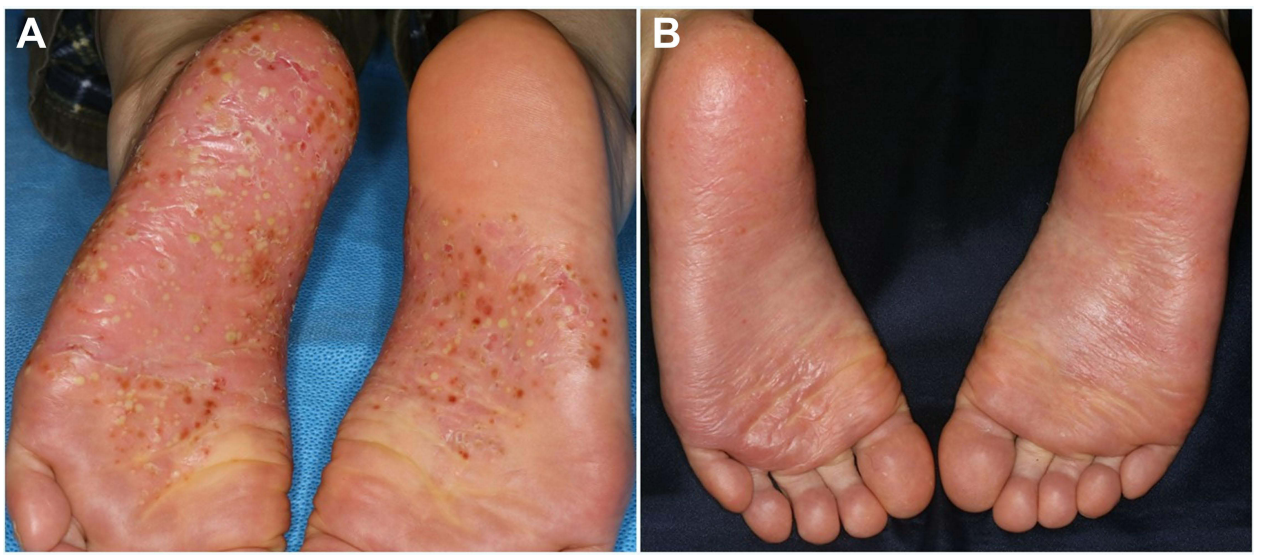

Figure 4 Clinical improvement of plantar PPP lesions before (A) and after (B) guselkumab therapy.

(73.3\%), as compared with the placebo group (47.4\%). Among the patients with MRI assessment of the anterior chest wall region, the proportions of patients in the guselkumab group with a $\geq 2$ grade improvement, 1 grade improvement, and no change were $3.4 \%, 27.6 \%$, and $65.5 \%$, respectively, at week 52 . The proportion of patients who had no or slight pain gradually increased, accounting for nearly $90 \%$ at week 52 .

Other biologics targeting IL-1 and IL-36 have been reported for PPP in a case series or clinical trials. ${ }^{55}$ Anakinra showed only a partial response. For PPP patients, spesolimab, a humanized monoclonal antibody against IL-36 receptor, was intravenously administered every 4 weeks at a dose of $900 \mathrm{mg}(\mathrm{n}=19)$ or $300 \mathrm{mg}$ $(\mathrm{n}=19)$ or placebo $(\mathrm{n}=21) .{ }^{56}$ At week 16 , the ratio achieving PPPASI50 among 6/19 (31.6\%) in each spesolimab group and 5/21 (23.8\%) in placebo group did not reach significant difference, and the primary endpoint was not met. Spesolimab was well tolerated, and adverse events were similar to those of placebo through the 32 observational period.

\section{Safety of Guselkumab}

The proportion of treatment-emergent adverse events, which were generally mild to moderate in severity, was comparable between the guselkumab and placebo groups. ${ }^{52,53}$ Frequent adverse effects included nasopharyngitis, headache, contact dermatitis, and injection site erythema. Serious adverse events were reported in 2 of the 25 patients receiving guselkumab; pyelonephritis in 1 patient and gastric cancer in the other. ${ }^{52}$ The frequency of infection was similar across both treatment groups. In the phase 3 trials, guselkumab was well-tolerated and had a favorable safety profile up to 52 weeks. ${ }^{53}$ Serious treatment-emerging adverse events were observed in 8 patients in total ( 2 in the placebo group and 6 in the guselkumab group), but no serious infections were reported. IL-23 inhibitors, unlike IL-17 inhibitors, do not aggravate intestinal inflammation. ${ }^{57}$ Moreover, IL-23 inhibitors have not been associated with an increased risk of candida infections. ${ }^{57}$

\section{Immunogenicity of Guselkumab}

In the phase 3 studies for moderate-to-severe plaque psoriasis, VOYAGE-1 and VOYAGE-2, anti-drug antibodies (ADA) in the sera were examined. Through up to 100 weeks of drug exposure, $8.5 \%$ (146/1713) of guselkumabtreated patients showed positive ADA; however, the ADA titers were low in most of the patients. ${ }^{58}$ Among the 146 ADA + patients, ADA was transiently detected in 66 patients $(45.2 \%)$. Serum guselkumab concentrations were comparable between the ADA+ and ADA- patients, and the development of ADA to guselkumab was not relevant to clinical efficacy or safety through week 100. In the 106 PPP patients treated with guselkumab, ADA was detected in 4 patients through week $52 .{ }^{53}$

\section{Management of PPP}

Because PPP is closely related with focal infection, smoking habit, and various comorbidities, it is important to educate patients to practice whatever they can do by themselves. For example, they must quit smoking, gargle to avoid catching a cold, reduce weights, and take care of dental conditions. Such patient education is important to manage skin and joint symptoms, in addition to medical treatment. PPP treatment is challenging, because topical therapies are usually not effective. Furthermore, many of the drugs are off-label use 


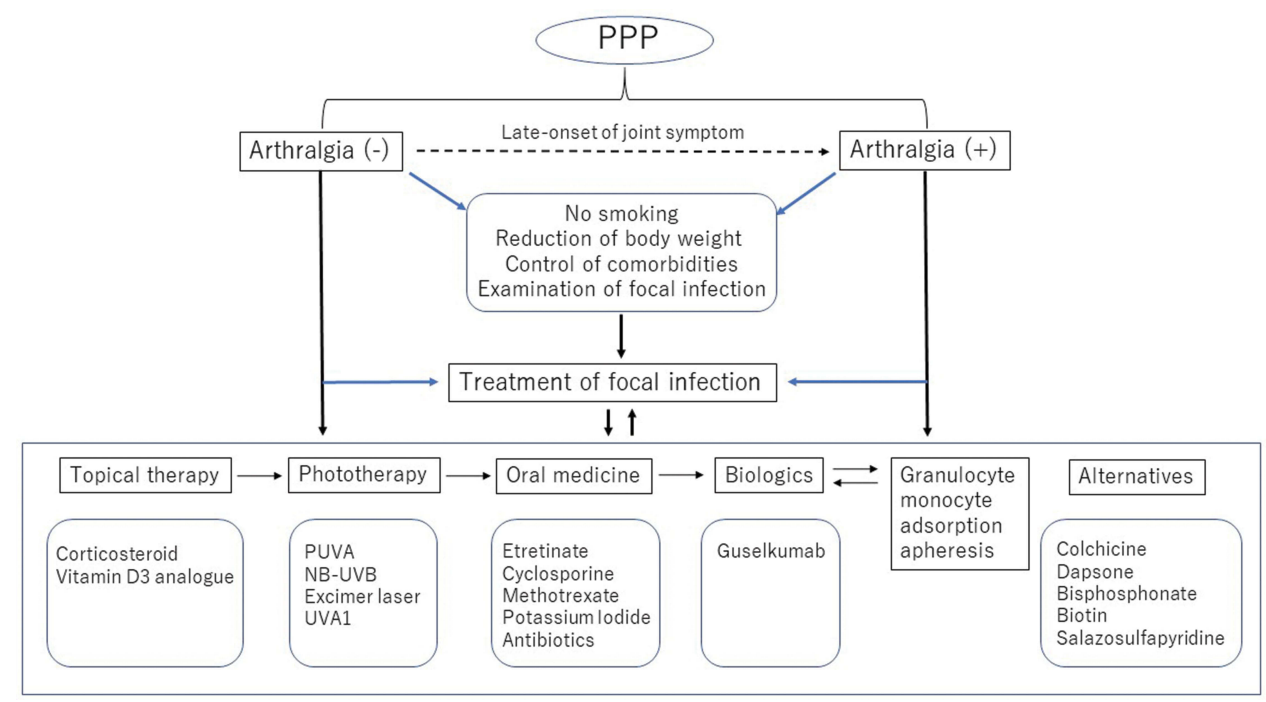

Figure 5 Management of Japanese PPP patients is shown. PPP patients are examined whether they have joint manifestation, and educated to quit smoking, reduce body weight, avoid triggers such as cold. Also, they should be checked for focal infection and comorbidities. Topical, systemic, and targeted immunomodulatory therapies, as well as phototherapy and granulocyte monocyte adsorption apheresis therapies are adopted.

and lack enough evidence; however, oral treatment with retinoids, cyclosporine, methotrexate, antibiotics, colchicine, potassium iodide, and itraconazole, as well as dapsone, bisphosphonate, and biotin is adopted. ${ }^{20}$ Adverse events due to long-term use of oral immunomodulatory and immunosuppressive drugs such as liver and renal dysfunction should be monitored. In addition, phototherapy and granulocyte monocyte adsorption apheresis have been used for refractory cases. ${ }^{20}$ For PAO, adequate pain management is required. Recently, targeted therapy is available for PAO. In Japanese case reports, guselkumab showed immediate effects on the severe joint pain, ${ }^{59-61}$ even if the long-term involvement of joint symptoms. ${ }^{60}$ An example of an algorithm for patient management is shown in Figure 5.

\section{Conclusion}

We here reported on the characteristics of Japanese PPP patients and our experience of current therapies mainly focusing on guselkumab. There are still few satisfactory therapies, and patient satisfaction is low. In Japan, guselkumab is currently the only available biologic, and the approved dosage of guselkumab for PPP is $100 \mathrm{mg}$ administration by subcutaneous injection at weeks 0 and 4 , followed by every 8 weeks thereafter. Either doseescalation or shortening of the administration intervals are not approved. Therefore, if the secondary failure occurs, there is no alternative, and switched biologics are off-label use. Further new therapies are expected to meet the unmet needs.

\section{Funding}

There is no funding to report.

\section{Disclosure}

The author reports no conflicts of interest in this work.

\section{References}

1. de Waal AC, van de Kerkhof PC. Pustulosis palmoplantaris is a disease distinct from psoriasis. J Dermatolog Treat. 2011; 22:102-105. doi:10.3109/09546631003636817

2. Brunasso AMG, Massone C. Psoriasis and palmoplantar pustulosis: an endless debate? J Eur Acad Dermatol Venereol. 2017;31:e335337. doi: $10.1111 /$ jdv. 14131

3. Misiak-Galazka M, Wolska H, Rudnicka L. Is palmoplantar pustulosis simply a variant of psoriasis or a distinct entity? J Eur Acad Dermatol Venereol. 2017;31:e342-343. doi:10.1111/jdv.14136

4. Misiak-Galazka M, Wolska H, Rudnicka L. What do we know about palmoplantar pustulosis? $J$ Eur Acad Dermatol Venereol. 2017;31:38-44. doi:10.1111/jdv.13846

5. Yamamoto T. Extra-palmoplantar lesions associated with palmoplantar pustulosis. J Eur Acad Dermatol Venereol. 2009;23(11):12 27-1232. doi:10.1111/j.1468-3083.2009.03296.x

6. Brunasso AMG, Massone C. Can we really separate palmoplantar pustulosis from psoriasis? J Eur Acad Dermatol Venereol. 2010;24:619-621. doi:10.1111/j.1468-3083.2010.03648.x

7. Yamamoto T. Can we really separate palmoplantar pustulosis from psoriasis? Reply. J Eur Acad Dermatol Venereol. 2010;24:621. doi:10.1111/j.1468-3083.2010.03649.x

8. Yamamoto T. Similarity and difference between palmoplantar pustulosis and pustular psoriasis. J Dermatol. 2021;48:750-760. doi:10. $1111 / 1346-8138.15826$

9. Uehara M, Ofuji S. The morphogenesis of pustulosis palmaris et plantaris. Arch Dermatol. 1974;109:518-520. doi:10.1001/archderm. 1974.01630040028005

10. Krieg PHG, Bacharach-Buhles M, el-Gammal S, Altmeyer P. The pustule in palmoplantar psoriasis: transformed vesicle or mature microabscess? Dermatology. 1992;185(2):104-112. doi:10.1159/000247423 
11. Engin B, Askin O, Tüzün Y. Palmoplantar psoriasis. Clin Dermatol. 2017;35:19-27. doi:10.1016/j.clindermatol.2016.09.004

12. Burden $\mathrm{AD}$, Kemmett $\mathrm{D}$. The spectrum of nail involvement in palmoplantar pustulosis. Br J Dermatol. 1996;134(6):1079-1082. doi:10.1111/j.1365-2133.1996.tb07946.x

13. Hiraiwa T, Yamamoto T. Nail involvement associated with palmoplantar pustulosis. Int J Dermatol. 2017;56(2):e28-29. doi:10.1111/ ijd. 13224

14. Masuda-Kuroki K, Murakami M, Kishibe M, et al. Diagnostic histopathological features distinguishing palmoplantar pustulosis from pompholyx. J Dermatol. 2019;46:399-408. doi:10.1111/1346-8138.14850

15. Yamamoto T. Pustulotic arthro-osteitis associated with palmoplantar pustulosis. J Dermatol. 2013;40:857-863. doi:10.1111/1346-8138.12272

16. Torii $\mathrm{H}$, Nakagawa $\mathrm{H}$, Ishibashi Y. Osteoarthritis in 84 Japanese patients with palmoplantar pustulosis. $J$ Am Acad Dermatol. 1994;31:732-735. doi:10.1016/S0190-9622(94)70233-0

17. Ishibashi A, Nishiyama Y, Endo M, et al. Orthopdic symptoms in pustular bacterid (pustulosis palmaris et plantaris): tietze's syndrome and arthritis of manubriosternal joint due to focal infection. J Dermatol. 1977;4:53-59. doi:10.1111/j.1346-8138.1977.tb01011.x

18. Ohashi T, Hiraiwa T, Yamamoto T. Low prevalence of anti-cyclic citrullinated peptide antibodies in Japanese patients with pustulotic arthro-osteitis. Indian J Dermatol. 2016;61:221-222. doi:10.4103/ 0019-5154.177769

19. Yamamoto T, Hiraiwa T, Tobita R, et al. Characteristics of Japanese patients with pustulotic arthro-osteitis associated with palmoplantar pustulosis: a multicenter study. Int J Dermatol. 2020;59:441-444. doi:10.1111/ijd.14788

20. Yamamoto T. Clinical characteristics of Japanese patients with palmoplantar pustulosis. Clin Drug Invest. 2019;39:241-252. doi:10.1007/s40261-018-00745-6

21. Harper EG, Guo C, Rizzo H, et al. Th17 cytokines stimulate CCL20 expression in keratinocytes in vitro and in vivo: implications for psoriasis pathogenesis. J Invest Dermatol. 2009;129:2175-2183. doi:10.1038/jid.2009.65

22. Yoshizaki T, Bandoh N, Ueda S, et al. Up-regulation of CC chemokine receptor 6 on tonsillar $T$ cells and its induction by in vitro stimulation with $\alpha$-streptococci in patients with pustulosis palmaris et plantaris. Clin Exp Immunol. 2009;157:71-82. doi:10.1111/j.13652249.2009.03945.x

23. Nozawa H, Kishibe K, Takahara M, Harabuchi Y. Expression of cutaneous lymphocyte-associated antigen (CLA) in tonsillar T-cells and its induction by in vitro stimulation with alpha-streptococci in patients with pustulosis palmaris et plantaris (PPP). Clin Immunol. 2005;116:42-53. doi:10.1016/j.clim.2005.01.009

24. Harabuchi Y, Takahara M. Pathogenic role of palatine tonsils in palmoplantar pustulosis: a review. J Dermatol. 2019;46:931-939. doi:10.1111/1346-8138.15100

25. Murakata H, Harabuchi Y, Kataura A. Increased interleukin-6, interferon-gamma and tumor necrosis factor-alpha production by tonsillar mononuclear cells stimulated with alpha-streptococci in patients with pustulosis palmaris et plantaris. Acta Otolaryngol. 1999;119:384-391. doi:10.1080/00016489950181431

26. Takahara M, Hirata $\mathrm{Y}$, Nagato $\mathrm{T}$, et al. Treatment outcome and prognostic factor of tonsillectomy for palmoplantar pustulosis and pustulotic arthro-osteitis: a retrospective subjective and objective quantitative analysis of 138 patients. J Dermatol. 2018;45:812-823. doi:10.1111/1346-8138.14348

27. Valeri M, Raffatellu M. Cytokines IL-17 and IL-22 in the host response to infection. Pathog Dis. 2016;74:ftw111. doi:10.1093/ femspd/ftw111

28. El-Zayadi AA, Jones EA, Churchman SM, et al. Interleukin-22 drives the proliferation, migration and osteogenic differentiation of mesenchymal stem cells: a novel cytokine that could contribute to new bone formation in spondyloarthropathies. Rheumatology. 2017; 56:488-493. doi:10.1093/rheumatology/kew384
29. Krishnan V, Bryant HU, MacDougald OA. Regulation of bone mass by Wnt signaling. J Clin Invest. 2006;116:1202-1209. doi:10.1172/ JCI28551

30. Chen G, Deng C, Li Y-P. TGF- $\beta$ and BMP signaling in osteoblast differentiation and bone formation. Int J Biol Sci. 2012;8:272-288. doi:10.7150/ijbs.2929

31. Kouno M, Nishiyama A, Minabe M, et al. Retrospective analysis of the clinical response of palmoplantar pustulosis after dental infection control and dental metal removal. J Dermatol. 2017;44:695-698. doi:10.1111/1346-8138.13751

32. Moutsopoulos NM, Kling HM, Angelov N, et al. Porphyromonas gingivalis promotes Th17 inducing pathways in chronic periodontitis. J Autoimmun. 2012;39:294-303. doi:10.1016/j.jaut.2012.03.003

33. Murakami M, Kaneko T, Nakatsuji T, et al. Vesicular LL-37 contributes to inflammation of the lesional skin of palmoplantar pustulosis. PLoS One. 2014;9(10):e110677. doi:10.1371/journal. pone. 0110677

34. Skov L, Beurskens FJ, Zachariae COC, et al. IL-8 as antibody therapeutic target in inflammatory diseases: reduction of clinical activity in palmoplantar pustulosis. J Immunol. 2008;181:669-679. doi:10.4049/jimmunol.181.1.669

35. Ozawa M, Terui T, Tagami H. Localization of IL-8 and complement components in lesional skin of psoriasis vulgaris and pustulosis palmaris et plantaris. Dermatology. 2005;211:249-255. doi:10.1159/ 000087019

36. Keller M, Spanou Z, Schaerli P, et al. T cell-regulated neutrophilic inflammation in autoinflammatory diseases. $J$ Immunol. 2005;175:7678-7686. doi:10.4049/jimmunol.175.11.7678

37. Eriksson MO, Hagforsen Lundin IP, Michaëlsson G. Palmoplantar pustulosis: a clinical and immunohistological study. $\mathrm{Br} J$ Dermatol. 1998;138:390-398. doi:10.1046/j.1365-2133.1998.02113.x

38. Murakami M, Ohtake T, Horibe Y, et al. Acrosyringium is the main site of the vesicle/pustule formation in palmoplantar pustulosis. J Invest Dermatol. 2010;130:2010-2016. doi:10.1038/jid.2010.87

39. Hagforsen E, Michaëlsson G, Stridsberg M. Normal and PPP-affected palmoplantar sweat gland express neuroendocrine markers chromogranins and synaptophysin differently. Arch Dermatol Res. 2010;302:685-693. doi:10.1007/s00403-010-1070-3

40. Hagforsen E. The cutaneous non-neuronal cholinergic system and smoking related dermatoses: studies of the psoriasis variant palmoplantar pustulosis. Life Sci. 2007;80:2227-2234. doi:10.1016/j.lfs.2007.01.045

41. Hagforsen E, Edvinsson M, Nordlind K, Michaëlsson G. Expression of nicotinic receptors in the skin of patients with palmoplantar pustulosis. Br J Dermatol. 2002;146:383-391. doi:10.1046/j.13652133.2002.04640.x

42. Kobayashi K, Kamekura R, Kato J, et al. Cigarette smoke underlies the pathogenesis of palmoplantar pustulosis via an IL-17A-induced production of IL-36 $\gamma$ in tonsillar epithelial cells. J Invest Dermatol. 2020;141(6):1533-1541.e4. doi:10.1016/j.jid.2020.09.028

43. Lillis JV, Guo C-S, Lee JJ, Blauvelt A. Increased IL-23 expression in palmoplantar psoriasis and hyperkeratotic hand dermatitis. Arch Dermatol. 2010;146(8):918-919. doi:10.1001/archdermatol.2010.168

44. Murakami M, Hagforsen E, Morhenn V, et al. Patients with palmoplantar pustulosis have increased IL-17 and IL-22 levels both in the lesion and serum. Exp Dermatol. 2011;20(10):845-847. doi:10.1111/ j.1600-0625.2011.01325.x

45. Hagforsen E, Hedstrand H, Nyberg F, Michaëlsson G. Novel findings of Langerhans cells and interleukin-17 expression in relation to the acrosyringium and pustule in palmoplantar pustulosis. Br J Dermatol. 2010;163(3):572-579. doi:10.1111/j.1365-2133.2010.09819.x

46. Morales-Múnera C, Vilarrasa E, Puig L. Efficacy of ustekinumab in refractory palmoplantar pustular psoriasis. Br J Dermatol. 2013;168 (4):820-824. doi:10.1111/bjd.12150

47. Gerdes S, Franke J, Domm S, Mrowietz U. Ustekinumab in the treatment of palmoplantar pustulosis. Br J Dermatol. 2010;163 (5):1116-1118. doi:10.1111/j.1365-2133.2010.09897.x 
48. Torre KM, Payette MJ. Combination biologic therapy for the treatment of severe palmoplantar pustulosis. JAAD Case Rep. 2017;3 (3):240-242. doi:10.1016/j.jdcr.2017.03.002

49. Shmidt E, Wetter DA, Ferguson SB, Pittelkow MR. Psoriasis and palmoplantar pustulosis associated with tumor necrosis factor- $\alpha$ inhibitors: the Mayo Clinic experience, 1998 to 2010. J Am Acad Dermatol. 2012;67(5):e179-185. doi:10.1016/j.jaad.2011.05.038

50. Benzaquen M, Flachaire B, Rouby F, et al. Paradoxical pustular psoriasis induced by ustekinumab in a patient with Crohn's diseaseassociated spondyloarthropathy. Rheumatol Int. 2018;38(7):12 97-1299. doi:10.1007/s00296-018-4034-0

51. Satoh M, Yamamoto T. Secukinumab-induced palmar vesiculopustular eruption in a patient with psoriasis. Dermatol Ther. 2020;33: e13332. doi:10.1111/dth. 13332

52. Terui T, Kobayashi S, Ohkubo Y, et al. Efficacy and safety of guselkumab, an anti-interleukin 23 monoclonal antibody, for palmoplantar pustulosis: a randomized clinical trial. JAMA Dermatol. 2018;154:309-316. doi:10.1001/jamadermatol.2017.5937

53. Terui T, Kobayashi S, Ohkubo Y, et al. Efficacy and safety of guselkumab in Japanese patients with palmoplantar pustulosis: a phase 3 randomized clinical trial. JAMA Dermatol. 2019;15 5:1153-1161. doi:10.1001/jamadermatol.2019.1394

54. Yamamoto T, Fukuda K, Morita A, et al. Efficacy of guselkumab in a subpopulation with pustulotic arthro-osteitis through week 52: an exploratory analysis of a phase 3, randomized, double-blind, placebo-controlled study in Japanese patients with palmoplantar pustulosis. J Eur Acad Dermatol Venereol. 2021;34:2318-2329. doi:10.1111/jdv.16355
55. Misiak-Galazka M, Zozula J, Rudnicka L. Palmoplantar pustulosis: recent advances in etiopathogenesis and emerging treatments. $\mathrm{Am}$ $J$ Clin Dermatol. 2020;21:355-370. doi:10.1007/s40257-020-00 503-5

56. Mrowietz U, Burden AD, Pinter A, et al. Spesolimab, an anti-interleukin-36 receptor antibody, in patients with palmoplantar pustulosis: results of a phase IIa, multicenter, double-blind, randomized, placebo-controlled pilot study. Dermatol Ther (Heidelb). 2021;11:571-585. doi:10.1007/s13555-021-00504-0

57. Yang EJ, Smith MP, Ly K, Bhutani T. Evaluating guselkumab: an anti-IL-23 antibody for the treatment of plaque psoriasis. Drug Des Devel Ther. 2019;13:1993-2000. doi:10.2147/DDDT.S137588

58. Zhu Y, Marini JC, Song M, et al. Immunogenicity of guselkumab is not clinically relevant in patients with moderate-to-severe plaque psoriasis. J Invest Dermatol. 2019;139:1830-1834. doi:10.1016/j. jid.2019.02.018

59. Kikuchi N, Yamamoto T. Rapid dramatic improvement of pustulotic arthro-osteitis by guselkumab in a patient with palmoplantar pustulosis: a real-world experience. Our Dermatol Online. 2020;11: 199-200. doi:10.7241/ourd.20202.26

60. Yamamoto T. Effects of guselkumab on ankylosing spondylitis-type pustulotic arthro-osteitis in a patient with palmoplantar pustulosis. Dermatol Ther. 2020;33:e14088. doi:10.1111/dth.14088

61. Ikumi N, Fujita H, Terui T. Guselkumab improves joint pain in patients with pustulotic arthro-osteitis: a retrospective pilot study. J Dermatol. 2021;48:199-202. doi:10.1111/1346-8138.15632
Clinical Pharmacology: Advances and Applications is an international, peer-reviewed, open access journal publishing original research, reports, reviews and commentaries on all areas of drug experience in humans. The manuscript management system is completely online and includes a very quick and fair peer-review system, which is all easy to use. Visit http://www.dovepress.com/testimonials.php to read real quotes from published authors. 